Mon. Not. R. Astron. Soc. 000, 000-000 (0000) Printed 10 March $2019 \quad$ (MN LATEX style file v2.2)

\title{
The Origin and Properties of Intracluster Stars in a Rich Cluster
}

\author{
Beth Willman $^{1 \star}$, Fabio Governato ${ }^{2,3} \dagger$, James Wadsley ${ }^{4}$ and Thomas Quinn ${ }^{2}$ \\ ${ }^{1}$ Center for Cosmology and Particle Physics, Department of Physics, New York University, 4 Washington Place, New York, NY 10003, USA \\ ${ }^{2}$ Department of Astronomy, University of Washington, Box 351580, Seattle, WA 98195, USA \\ ${ }^{3}$ Osservatorio Astronomico Di Brera, via Brera 28, Milano, Italy \\ ${ }^{4}$ Department of Physics and Astronomy, McMaster University, Hamilton, Ontario L88 4M1, Canada
}

10 March 2019

\begin{abstract}
We use a multi million particle N-body $+\mathrm{SPH}$ simulation to follow the formation of a rich galaxy cluster in a $\Lambda \mathrm{CDM}$ cosmology, with the goal of understanding the origin and properties of intracluster stars. The simulation includes gas cooling, star formation, the effects of a uniform ultraviolet background and feedback from supernovae. Halos that host galaxies as faint as $\mathrm{M}_{R}=-19.0$ are resolved by this simulation, which includes $85 \%$ of the total galaxy luminosity in a rich cluster. We find that the accumulation of intracluster light (ICL) is an ongoing process, linked to infall and stripping events. The unbound star fraction varies with time between $10 \%$ and $22 \%$ of the total amount of cluster stars, with an overall trend to increase with time. The fraction is $20 \%$ at $\mathrm{z}=0$, consistent with observations of galaxy clusters. The surface brightness profile of the $\mathrm{cD}$ shows an excess compared to a de Vaucouleur profile near 200 $\mathrm{kpc}$, which is also consistent with observations. Both massive and small galaxies contribute to the formation of the ICL, with stars stripped preferentially from the outer, lower metallicity, parts of their stellar distributions. Simulated observations of planetary nebulae (PNe) show significant substructure in velocity space, tracing separate streams of stripped intracluster stars. Despite an unrelaxed distribution, individual intracluster PNe might be useful mass tracers if more than 5 fields at a range of radii have measured line-of-sight velocities, where an accurate mass calculation depends more on the number of fields than the number of PNe measured per field. However, the orbits of IC stars are more anisotropic than those of galaxies or dark matter, which leads to a systematic underestimate of cluster mass relative to that calculated with galaxies, if not accounted for in dynamical models. Overall, the properties of ICL formed in a hierarchical scenario are in good agreement with current observations, supporting a model where ICL originates from the dynamical evolution of galaxies in dense environments. ICL should thus be ubiquitous in galaxy clusters.
\end{abstract}

Key words: galaxies: formation

\section{INTRODUCTION}

For many years, the presence of intracluster (IC) stars has been well documented by observations of diffuse light between cluster galaxies (e.g. Zwickv 1951; Gonzalez et al. 2000; Feldmeier et al. 2002; Feldmeier et al. 2004; Zibetti \& White 2004; Krick et al. 2004). Recently, numerous new searches for IC stars have uncovered individual stars between cluster galaxies (PNe: Theuns \& Warren 1997; Arnaboldi et al. 2002; Arnaboldi et al. 2003;

\footnotetext{
* Email: willman@astro.washington.edu $\dagger$ Brooks Fellow
}

Feldmeier et al. 1998, 2003; Okamura et al. 2002; red giants: Durrell et al. 2002; SNeIa: Gal-Yam et al.|2003), and individual tidal streams in clusters (Trentham \& Mobasher 1998; Gregg \& West 1998; Calcáneo-Roldán et al. 2000; Feldmeier et al. 2002). These studies, and others, all measure an amount of intracluster light that is between $10 \%$ and $50 \%$ of the total cluster luminosity, suggesting that intracluster stars are a generic feature of galaxy clusters.

The observable properties of intracluster stars are likely closely linked to the dynamical history of galaxy clusters, as IC stars are a natural by-product of cluster evolution in hierarchical models such as $\Lambda$ CDM. Numerical simulations have demonstrated that the dynamical evolution of 
galaxies in clusters is mainly dictated by global gravitational tides and fast encounters between galaxies that alter their morphologies and strip them of their dark matter halos (Moore et al. 1996; Dubinski 1998; Ghigna et al. 1998; Calcáneo-Roldán et al. 2000; Maver et al.|2001). For example, Moore et al. (1996) studied the dynamical evolution of individual galaxies inside a realistic cluster potential to show that, depending on their internal structure, they might undergo significant stripping of their stellar material. As galaxies get stripped, stars in their tidal streams carry memory of the parent galaxy's orbit for several dynamical times. Spatial streams seen in Hydra and Coma have thus been used to trace past dynamical interactions (Calcáneo-Roldán et al. 2000).

As stripped stars become mixed into the global cluster potential, they still bear signatures of the cluster's accretion and dynamical history, although the extent of this is uncertain. Thus far, numerical studies that addressed such uncertainties have focused on the $\mathrm{z}=0$ properties of intracluster stars. Dubinski (1998) used an approach where disk/halo galaxy models were substituted for dark matter halos in a cluster simulation in a critical universe to show that a significant fraction of stars end up unbound to individual galaxies. Napolitano et al. (2003) compared a collisionless N-body simulation of the formation of a galaxy cluster with recent observations of Virgo IC PNe. A simple scheme to identify a diffuse stellar component in the simulation showed that intracluster stars are largely unrelaxed in velocity and are clustered on $50 \mathrm{kpc}$ scales at 500 $\mathrm{kpc}$ from the cluster center, in good agreement with the observed clustering properties of the diffuse population of PNe in Virgo. Recently, Sommer-Larsen et al. (2004) looked at the $\mathrm{z}=0$ photometric properties and metallicity distribution of IC stars in a cosmological simulation, and found them in good agreement with observations. Murante et al. (2004) studied clusters in a large cosmological simulation and found fractions of unbound cluster stars ranging from 10 - 50\%. However, both Sommer-Larsen et al. (2004) and Murante et al. (2004)'s spatial resolution was much coarser than the average scale size of cluster galaxies, which should overproduce intracluster stars, due to overly efficient stripping.

The numerous remaining uncertainties in the expected properties of IC stars leave much room for improvement in this burgeoning field of study. For example, no consensus exists on when and how many IC stars should actually be produced in a case where the entire population of a forming galaxy cluster is realistically simulated. Nor is there consensus on whether IC stars originate mostly from the stripped outer parts of bright galaxies or rather from a population of tidally destroyed low surface brightness galaxies. It is also unclear whether observed cluster to cluster variations in measured IC fractions and radial profiles are due to different cluster formation histories and dynamical states, rather than a cosmic scatter in the expected properties of IC stars.

Furthermore, little has been done theoretically to predict the dynamics of the intracluster population; there is only one published analysis of the velocity distribution of IC PNe (Sommer-Larsen et al. 2004). Positions and velocities have been measured for hundreds of IC planetary nebulae in Virgo (e.g. Feldmeier et al. 2003; Arnaboldi et al. 2002) making it possible to use PNe to measure cluster mass, as
ICPNe outnumber cluster galaxies (Ford et al. 2002). However, it is unclear if an accurate mass measurement with $\mathrm{PNe}$ is feasible, due to the substructure found in observations and simulations of IC stellar populations.

In this paper, we address the outstanding questions above by using a simulation of a rich galaxy cluster in a realistic cosmological environment by using an unsurpassed coupling of high spatial and mass resolution. This allows us to follow the star formation histories of individual galaxies as faint as $L_{*} / 10$ for the first time, a crucial requirement to resolve the formation of the large majority of the stars formed in the cluster. Nearly $90 \%$ of galaxy light in the Coma cluster resides in galaxies with $\mathrm{L}>\mathrm{L}_{*} / 10$ (Mobasher et al. 2003). Also, due to a spatial resolution higher than most previous analyses, our simulations produced a realistic distribution of galaxy sizes as a function of stellar mass and dark matter halo mass. This requirement is fundamental for a faithful description of the stripping processes in the dense cluster environment. Also, although simulations with a more ad hoc approach to tracing stars produce worthwhile results, a simulation that includes star formation produces a more accurate model of ICL as a continuous, ongoing process.

The aims of this paper are: a) to shed light on the uncertain origin and properties of intracluster stars in a $\Lambda \mathrm{CDM}$ Universe and how they relate to the dynamical state of the cluster and b) test their use as dynamical tracers of a galaxy cluster potential. In $\S 2$, we describe the simulation; in $\S 3$, we describe the origin and evolution of the IC population; in $\S 4$, we discuss IC stars as tracers of the cluster's dynamical history; and in $\S 5$, we evaluate the utility of IC PNe as tracers of cluster mass.

\section{THE SIMULATION}

\subsection{GASOLINE}

We selected a candidate Coma cluster from an existing low resolution, dark matter only simulation in a concordance $\left(\Lambda=0.7, \Omega_{0}=0.3, \sigma_{8}=1\right)$ cosmology and resimulated it at higher resolution using the volume renormalization technique (Katz \& White 1993). To perform this simulation, we used GASOLINE (Wadslev et al. 2004), a smooth particle hydrodynamic (SPH), parallel treecode that is both spatially and temporally adaptive. The version of GASOLINE we used implemented i) Compton and radiative cooling, ii) star formation and 'minimal' supernova feedback as described by Katz et al. (1996), and iii) a UV background following Haardt \& Madau (1996). The opening angle, $\theta$, was 0.5 until $\mathrm{z}=2$ and 0.7 thereafter, and the time-stepping criterion, $\eta$, was 0.2, as in Diemand et al. (2004).

We ran four different simulations of the same cluster:

- C1 is a dark matter only run that we use to evaluate our halo completeness limit. This is a crucial measure as stars can form in poorly resolved halos but should not be included into our analysis.

- C2 is our fiducial run that includes gas cooling processes, star formation and SN feedback. The high resolution region of this simulation contains dark and star particles of $1.5 \times 10^{9}$ and $6.7 \times 10^{7} M_{\odot}$ respectively, with a force softening of $3.75 \mathrm{kpc}$. There are $2 \times 10^{6}$ particles within the cluster's virial radius at $\mathrm{z}=0$. We will discuss results from $\mathrm{C} 2$, unless noted otherwise. 


\begin{tabular}{cccccc}
\hline run & $\begin{array}{c}\mathrm{N}_{\text {dark }} \\
\text { within }\end{array}$ & $\begin{array}{c}\mathrm{N}_{\text {star }} \\
\text { vir }\end{array}$ & \multicolumn{2}{c}{$\begin{array}{c}\mathrm{M}_{\text {dark }} \\
\mathrm{M}_{\odot} \text { per particle }\end{array}$} & $\begin{array}{c}\mathrm{M}_{\text {star }} \\
\mathrm{kpc}\end{array}$ \\
\hline $\mathrm{C} 1$ & 680814 & - & $1.7 \cdot 10^{9}$ & - & 3.75 \\
\hline $\mathrm{C} 2$ & 688754 & 868995 & $1.5 \cdot 10^{9}$ & $7.2 \cdot 10^{7}$ & 3.75 \\
\hline C2low & 85570 & 143326 & $1.2 \cdot 10^{10}$ & $8.3 \cdot 10^{8}$ & 7.5 \\
\hline $\mathrm{C} 3$ & 679574 & 516261 & $1.5 \cdot 10^{9}$ & $7.2 \cdot 10^{7}$ & 3.75 \\
\hline
\end{tabular}

Table 1. Simulation Parameters

- C2low is a lower resolution version of $\mathrm{C} 2$ with 8 times fewer particles.

- C3 is of equal resolution to $\mathrm{C} 2$, but includes the effects of additional feedback introduced by setting the gas at an entropy larger than $5 \mathrm{keV} \mathrm{cm}^{2}$ at $\mathrm{z}=3$ to about $50 \mathrm{keV} \mathrm{cm}^{2}$, as measured by X-ray observations of clusters and groups (Borgani et al. 2002).

All four clusters have $\mathrm{R}_{200}$, the radius enclosing an average $\delta \rho / \rho=200$, of $2.2 \mathrm{Mpc}, \mathrm{M}_{200} \sim 1.2 \cdot 10^{15} \mathrm{M}_{\odot}$, and a $1 \mathrm{D}$ velocity dispersion of $1000 \mathrm{~km} \mathrm{sec}^{-1}$. These are similar to the observed properties of the Coma cluster Hughes 1989).

Table 2.1 gives the number of particles, particle masses, and force softenings of each simulation. The stellar masses given in the table are the maximum mass of a star within each $\mathrm{z}=0$ cluster.

\subsection{Simulated Cluster Properties}

Figure 1 shows the spatial distribution of the cluster stars at $\mathrm{z}=0$, represented by a random selection of $2 \%$ of all stars. The clear asymmetry in this distribution suggests that the galaxy population is unrelaxed, which we show in $\S 3$ to be the case, due to several major accretion events at low redshift. The outlined black squares show the size of a $0.5 \mathrm{x}$ $0.5 \mathrm{deg}^{2}$ field at $15 \mathrm{Mpc}$, the distance of Virgo. We discuss the properties of stars in these fiducial fields in $\S 4$. There are two large groups in the cluster: Group One is a Virgo sized group that entered the cluster $1-2$ Gyr ago. Group Two is the size of Fornax, and entered the cluster 5 Gyr ago.

$20 \%$ of the simulated cluster's stars are unbound to individual galaxies at $\mathrm{z}=0$, consistent with the values of $10-$ $50 \%$ suggested by observations (see $\S 3.3$ for additional discussion). To distinguish stars gravitationally bound to individual galaxies from free-floating stars, we used the SKID halo finding algorithm on the gas and dark matter. We initially ran SKID with a large linking length of $18 \mathrm{kpc}$, to minimize identifying loosely bound stars at $\mathrm{z}=0$ as unbound. Because that choice of linking length misses a few halos within the $\mathrm{cD}$ envelope, we also ran SKID with a smaller linking length of $9 \mathrm{kpc}$ and included the additional galaxies in our sample. Based on a visual evaluation of the data, this combination produced the most robust identification of halos at $\mathrm{z}=0$. However, we found that our choice of linking length does not substantially affect the results. This procedure identified 650 galaxies within $\mathrm{R}_{200}$.

Due to the weak implementation of feedback in the simulations, star formation in dense gas regions proceeds unimpeded, resulting in a stellar baryon fraction of $36 \%$,

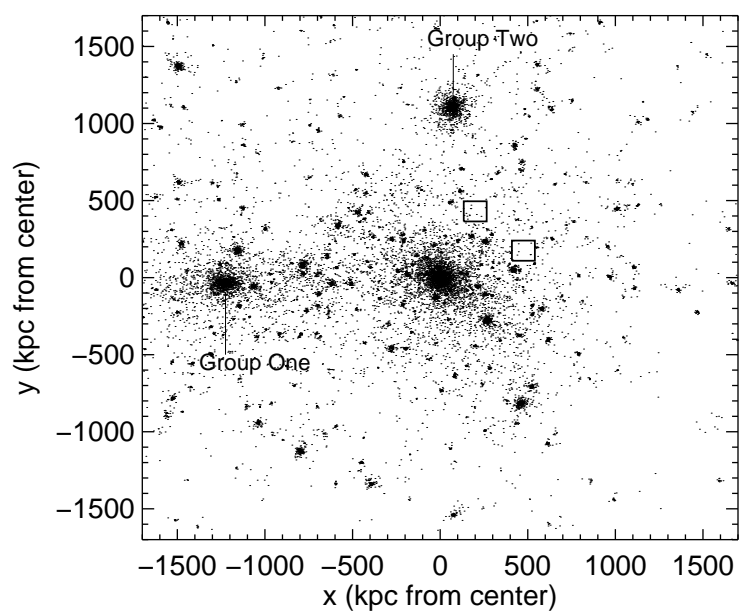

Figure 1. The spatial distribution of stars in the simulated Coma-like galaxy cluster, C2. Only $2 \%$ of the star particles are displayed, for clarity. The outlined squares show the size of a $0.5 \mathrm{x}$ $0.5 \mathrm{deg}^{2}$ field for the cluster at a distance of $15 \mathrm{Mpc}$, the distance of Virgo.

several times higher than the $6-12 \%$ suggested by observations of galaxy clusters (Bell et al. 2003; Balogh et al. 2001; Cole et al. 2001). This "cooling crisis" is a well known weakness of the current generation of cosmological SPH simulations (Cole et al. 2001, Bell et al. 2003, Balogh et al. 2001, Kay 2001). Producing the correct amount of stars in a cosmological simulation is a problem that depends critically on numerical resolution and on the poorly known details of star formation and feedback from SNe (Thacker \& Couchman 2000, Borgani et al. 2001), although see Springel \& Hernquist (2003) and Sommer-Larsen et al. (2004) for recent improvements.

However, this overproduction of stars does not adversely affect our results because star particles in $\mathrm{C} 2$ galaxies have a realistic distribution, and are thus accurate tracers of the dynamically stripped stellar population. If the simulated stellar halos are too compact or too diffuse, then the stripping efficiency by dynamical processes in the cluster would be under or overestimated, respectively. To determine the stellar distributions of galaxies in $\mathrm{C} 2$, we fit de Vaucouleurs effective radii $\left(R_{e}\right)$ to the 389 cluster galaxies with stellar mass $>M_{\text {limit }}\left(10^{9.9} M_{\odot}\right) . M_{\text {limit }}$ is the typical simulated stellar mass contained in a halo just above the conservative resolution limit of the simulation. We determine the halo resolution limit by a comparison with $\mathrm{C} 1$, the dark matter only run. The circular velocity function of $\mathrm{C} 1$ started to flatten $\sim v_{c}=100 \mathrm{~km} \mathrm{sec}^{-1}$, although C2's did not flatten until $70 \mathrm{~km} \mathrm{sec}^{-1}$. Halos less massive than $100 \mathrm{~km} \mathrm{sec}^{-1}$ would suffer less efficient cooling and may be incomplete due to numerical effects. We thus selected $\mathrm{v}_{c}=100 \mathrm{~km} \mathrm{sec}^{-1}$ as a conservative resolution limit to be confident of completeness in our analysis. Nearly all galaxies with stellar masses greater than $10^{9.9} M_{\odot}$ are hosted by halos with $\mathrm{v}_{c}>100 \mathrm{~km}$ $\mathrm{sec}^{-1}$. Halos more massive than this limit contain $98 \%$ of the simulated stellar mass. We cut on stellar mass, rather than velocity or dark matter mass, to facilitate matching 


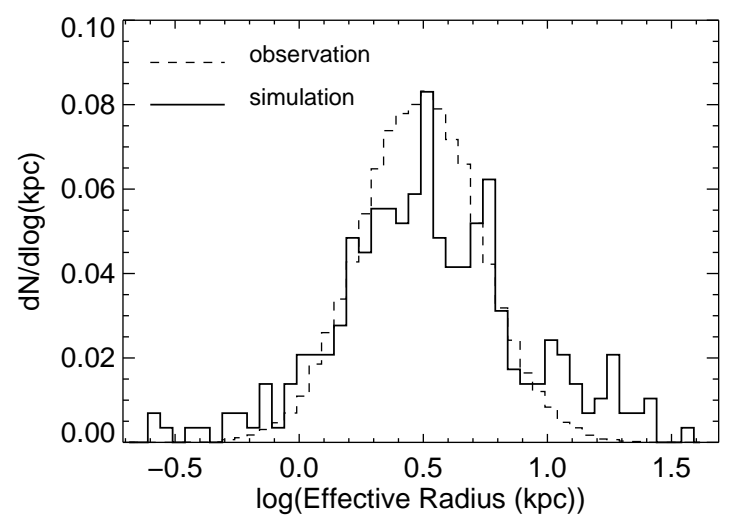

Figure 2. Histogram comparison of the distribution of de Vaucouleurs effective radii for our simulated galaxies vs the distribution measured by Bernardi et al. (2003) for 9000 early type galaxies in the SDSS.

each simulated halo to a galaxy drawn from a Coma cluster luminosity function ( $\$ 2.2 .1)$.

Figure 2 shows the resulting distribution of the simulated galaxies' effective radii, overplotted with the distribution measured by Bernardi et al. (2003), for 9000 early type galaxies in the SDSS. The $R_{\text {eff }}$ distribution of $\mathrm{C} 2$ galaxies is indeed in the same range and peaks at the same scale size as that observed by Bernardi et al. (2003). Although there is some excess high $R_{e}$ galaxies in the simulation relative to the observations, our measured distribution of scale sizes is a substantial improvement over existing studies of IC stars. Therefore, we conclude that our simulated galaxies have sufficiently realistic stellar profiles to be used to study the production of intracluster stars in a rich cluster.

\subsubsection{The Galaxy Luminosity Function}

One of our main goals is to study what fraction of ICL originates from galaxies of different masses. Our simulation resolved a range of 4 decades in stellar mass; to determine how far down the galaxy luminosity function (LF) this stellar mass range includes and to evaluate which of our simulations to focus this study on, we compare the luminosity functions of our simulations with that observed for the Coma cluster. Figure 3 shows a comparison between the original LFs of C2, C2low, C3, and the luminosity function for the entire Coma cluster as observed by Mobasher et al. (2003). To convert the total stellar mass of each simulated cluster galaxy to an $\mathrm{R}$ magnitude, we combine the Secker et al. (1997) observed R vs. B-R relationship for galaxies in Coma with the Bell \& de Jong (2001) $(\mathrm{M} / \mathrm{L})_{R}$ vs $(\mathrm{B}-\mathrm{R})$ relation with a scaled Salpeter IMF and Cole et al. (2000) galaxy evolution model.

As star particles formed in the simulation act as dynamical tracers, it is necessary that our simulation has a galaxy LF as close as possible to that observed for cluster galaxies. Figure 3 shows that $\mathrm{C} 2$ galaxies approximately follow a Schecter function, although the brightest galaxies overproduce stars. However, the lower resolution run, C2low, neither produces the correct number of stars in massive ha-

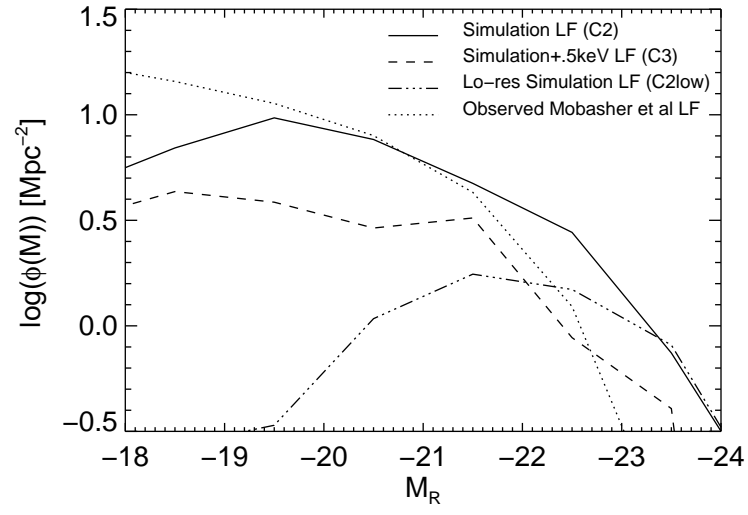

Figure 3. Original luminosity functions of simulated cluster galaxies in three different runs: low resolution, high resolution, and high resolution with an entropy injection of $0.5 \mathrm{keV}$ at $\mathrm{z}=$ 3 . For reference, the observed luminosity function of Mobasher et al (2003) is overplotted.

los nor resolves dark matter halos smaller than those that host $\mathrm{M}_{R}^{\star}$ galaxies. A run that resolves such a small fraction of the luminosity function would provide a very unrealistic and incomplete description of intracluster star accumulation. Although C3, the run with $0.5 \mathrm{keV}$ particle ${ }^{-1}$ energy injection, produces a reasonable luminosity function above $\mathrm{M}_{R}^{\star}$, the formation of galaxies fainter than $\mathrm{M}_{R}^{\star}$ is unrealistically suppressed. This feedback recipe, while useful to study the properties of the IGM (Borgani et al. 2002), is too crude to accurately simulate galaxy formation within the cluster. Based on these comparisons, we choose to focus our analysis only on $\mathrm{C} 2$.

To make our results directly comparable with observations, we derive corrected stellar masses and $\mathrm{R}$ magnitudes for each cluster galaxy by normalizing to the observed Mobasher et al. (2003) luminosity function (LF). Although the mass-luminosity relation of $\mathrm{C} 2$ is not correct, the basic correlation is correct: more stars form in more massive halos. Since the correlation between stellar and halo mass is monotonic, and we have a large number of halos, we are justified in using the observed luminosity function of the Coma cluster to calibrate the results of our star formation algorithm. We perform the normalization based on the 389 galaxies above the halo resolution limit (see above). The integrated Mobasher et al. (2003) LF of the entire Coma cluster contains 389 galaxies brighter than $\mathrm{R}=-19.0$, which includes $86 \%$ of the total light they observed in cluster galaxies. We thus create rank ordered populations of 389 galaxies drawn from a Mobasher LF cutoff at $\mathrm{R}=-19.0$, which is 2.8 magnitudes fainter than $\mathrm{M}_{R}^{\star}$ of Coma galaxies. We convert their $\mathrm{M}_{R} \mathrm{~S}$ to estimated stellar masses by combining Secker et al. (1997)'s observed color-magnitude relation for Coma with the Bell \& de Jong (2001) color-(M/L) relation used above. We then fit a function relating these stellar masses to the rank ordered simulation stellar masses. We convert $\mathrm{M}_{R}$ to stellar mass, rather than just matching simulated stellar mass to $\mathrm{M}_{R}$, so that we can normalize the mass of each individual star in the simulation. Figure 4 shows the normalized 


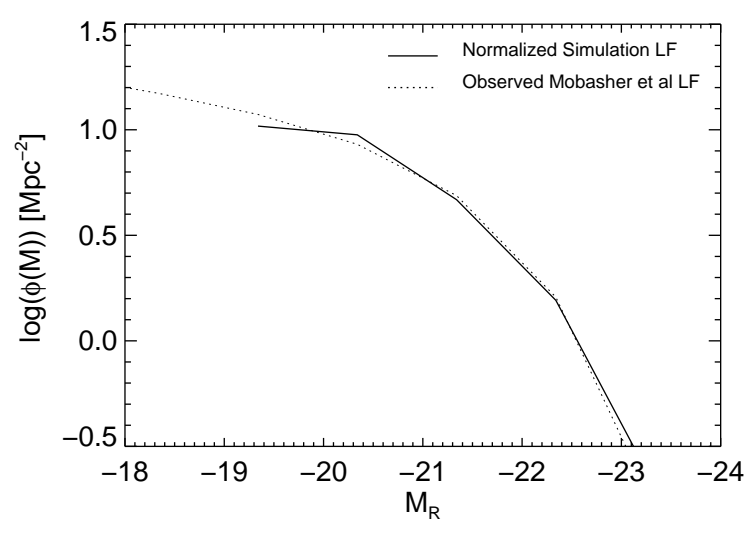

Figure 4. The luminosity function of simulated cluster galaxies after normalization, as compared to the observed luminosity function of Mobasher et al (2003).

luminosity function of our cluster. After normalizing, $10 \%$ of cluster baryon mass is in stars, vs. $36 \%$ pre-normalization.

We used the stellar mass normalization derived at $\mathrm{z}=0$ for cluster galaxies at all redshifts up to $\mathrm{z}=1.1$, correcting the derived luminosities for passive evolution.

\section{ORIGIN AND EVOLUTION OF THE INTRACLUSTER STARS}

Because this cosmological simulation includes a large dynamic range of galaxies, we can derive the contribution of galaxies to the IC population, as a function of their absolute magnitude. In this section, we determine the origin of IC stars and use our result to predict and interpret observed colors and metallicities of IC stars. We also determine the evolution of the intracluster star fraction and evaluate the extent to which it is a function of the cluster's dynamical state.

\subsection{Determining the Progenitor Halos of the Intracluster Stars}

We traced all of the stars unbound at $\mathrm{z}=0$ back until the epoch when they were last bound to a galaxy. As $85 \%$ of unbound stars were stripped since $\mathrm{z}=1.1$, we chose to only trace to that epoch. To reduce errors in assigning stars to their progenitor halos, we $(i)$ do not allow stars to be "stripped" from the cD galaxy, but rather traced them back to their original halos and ( $i i)$ require that a star is bound to its progenitor halo for at least two consecutive simulation outputs. We normalize the mass of each stripped star by the same factor that its progenitor's mass was normalized, as determined by the technique described in $\S 2.2 .1$. We use these normalized masses in the subsequent results presented in this paper.

\subsection{Determining the $\mathrm{z}=0$ Luminosities of the Progenitor Halos}

Thus far, observational studies of IC starlight have been limited to relatively nearby, low redshift, clusters. Therefore, we determine the $\mathrm{z}=0$ luminosity of the galaxy each progenitor evolves into. We trace the member stars of each progenitor galaxy forward in time to determine the halos they reside in at $\mathrm{z}=0$. We define the $\mathrm{z}=0$ galaxy, $\mathrm{G}_{f}$, of each progenitor, $\mathrm{G}_{i}$, as the $\mathrm{z}=0$ galaxy that contains the highest fraction of $\mathrm{G}_{i}$ 's stars. If the majority of $\mathrm{G}_{i}$ 's stars are unbound at $\mathrm{z}=$ 0 , then $\mathrm{G}_{f}$ must contain $\geq 40 \%$ of $\mathrm{G}_{i}$ 's 10 most bound stars. If not, the progenitor halo is considered to be destroyed by $\mathrm{z}=0$. Only $1 \%$ of unbound stars from galaxies brighter than $\mathrm{M}_{R}=-19$, were stripped from galaxies that were ultimately completely disrupted, and $9 \%$ of unbound stars were stripped from galaxies that ultimately merged with the $\mathrm{cD}$.

\subsection{Evolution}

Observations of diffuse intracluster light have suggested that the majority of the IC star population in some clusters was accumulated early in the formation of the cluster (Gonzalez et al. 2000). Authors have also suggested that the fraction of unbound stars in galaxy clusters may be linked to the dynamical state of the cluster (Feldmeier et al. 2002). In this section, we compare these past results to the evolution and fraction of intracluster stars in $\mathrm{C} 2$.

Figure [5] shows the evolution of C2's intracluster population back to $\mathrm{z}=1.1$. The top panel compares the growth rate of the intracluster stars with the overall cluster growth rate. Stars are added to the intracluster halo via stripping processes within the cluster environment and via the infall of large galaxy groups that already contain unbound stars. The growth of the IC population and the cluster as a whole are clearly linked and primarily occur by an ongoing series of events, rather than by a steady process. The peaks at $\mathrm{z}$ $=0.55$ and $\mathrm{z}=0.2$ to 0.1 correspond to the infall of large groups, ranging in size from Fornax to Virgo.

The bottom panel of Figure 5 shows the fraction of cluster stars that are unbound as a function of time. The IC star fraction in $\mathrm{C} 2$ displays a trend to increase with time. This trend is due to the continuous stripping of stars from cluster galaxies as they orbit. However, the fraction is not clearly linked to the dynamical state of the cluster. For example, the cluster underwent a substantial accretion event at $\mathrm{z}=.55$, but did not have an enhanced IC fraction as a result. Since $\mathrm{z}=1.1$, the IC fraction varied between a minimum of $10 \%$ and a maximum of $22 \%$, which closely matches the range in IC star fractions estimated for Virgo and several Abell clusters (Feldmeier et al. 1998; Feldmeier et al. 2004), although is slightly less than the fraction estimated for Coma (Bernstein et al. 1995) and a rich Abell cluster (Feldmeier et al. 2002). This difference could be due to the fact that the fraction we measure represents a lower limit to the fraction of cluster stars that are unbound, because can not include stars stripped from galaxies fainter than -19 in our analysis.

Although stars are continuously added to the intracluster population, the simulated cluster accumulated $50 \%$ of its IC stars within the last 2 Gyr. The upper panel of Figure 5 shows that this recent increase in the number of IC stars 


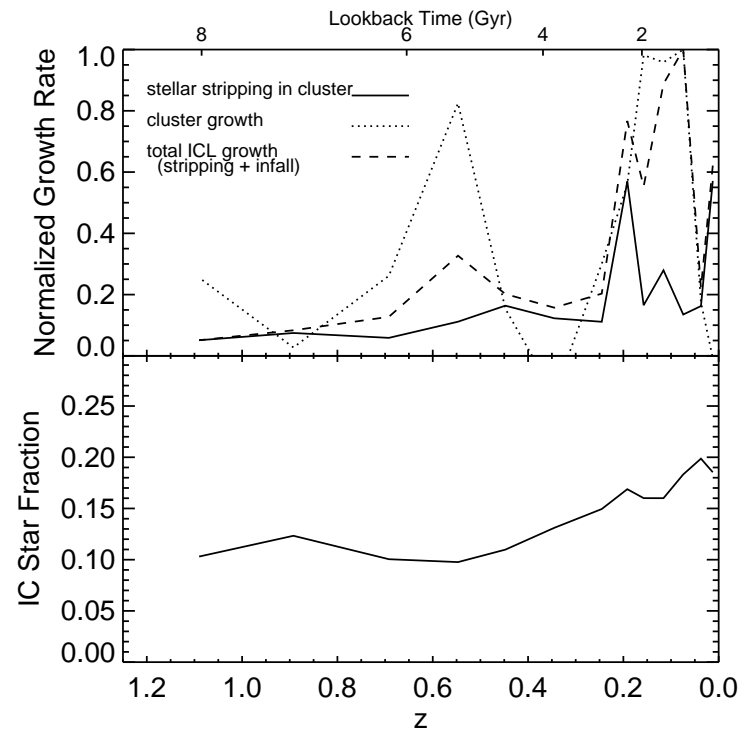

Figure 5. Top panel: Time evolution of the stripping and cluster growth rates compared with the time evolution of the fraction of cluster stars in the ICM. The values of stellar stripping and total ICL growth are both normalized to the maximum ICL growth rate. The values of cluster growth rate are normalized to the maximum cluster growth rate. Bottom panel: The time evolution of the unbound star fraction.

was connected to a substantial accretion event experienced by the cluster between 1 and 2 Gyr ago. The mean dynamical time of this cluster is $(3 \pi / 16 G \rho)^{-1 / 2} \sim 2$ Gyr, thus the cluster is not in a dynamically relaxed state, as suggested by the asymmetric spatial distribution of the cluster seen in Figure 1. The variation of IC fraction with time in just one cluster suggests that there is no universal IC fraction.

\subsection{Origin}

Figure 6] shows the fraction of IC stars stripped as a function of the absolute magnitude of their progenitor halo, both at $\mathrm{z}_{\text {strip }}$ and at $\mathrm{z}=0.9 \%$ of stars are stripped from galaxies that become fainter than -19 by $z=0$. The cumulative fraction of stars bound to halos with $\mathrm{M}_{R}<\mathrm{R}$ at $\mathrm{z}=0$ is also overplotted. The fact that the fraction of stars from halos with $\mathrm{M}_{R}<\mathrm{R}$ at the time of stripping closely follows the fraction of stars contained in halos (plus an offset in $\mathrm{M}_{R}$ ) shows that stripping efficiency is roughly constant with stellar mass. The offset in $M_{R}$ results from our accounting for passive evolution when determining $\mathrm{M}_{R}$ at $\mathrm{z}>0$. The $\mathrm{z}=0$ progenitor $\mathrm{M}_{R}$ distribution has a different shape than the $\mathrm{z}$ $=\mathrm{z}_{\text {strip }}$ distribution because stripped galaxies that are less massive than $\mathrm{M}^{*}$ often merge into more massive galaxies.

We find that massive galaxies contribute substantially to the IC population. Figure 6 also shows that $50 \%$ of freefloating cluster stars were stripped from progenitor galaxies with luminosities of $\mathrm{M}_{R}^{\star}$ or brighter. Because stars are preferentially stripped from the outer regions of galaxies, IC stars should bear the properties of stars in the outskirts of galaxies from which they were stripped. Assuming passive evolution, these should be similar to the $\mathrm{z}=0$ properties of stars in the outskirts of progenitor galaxies.

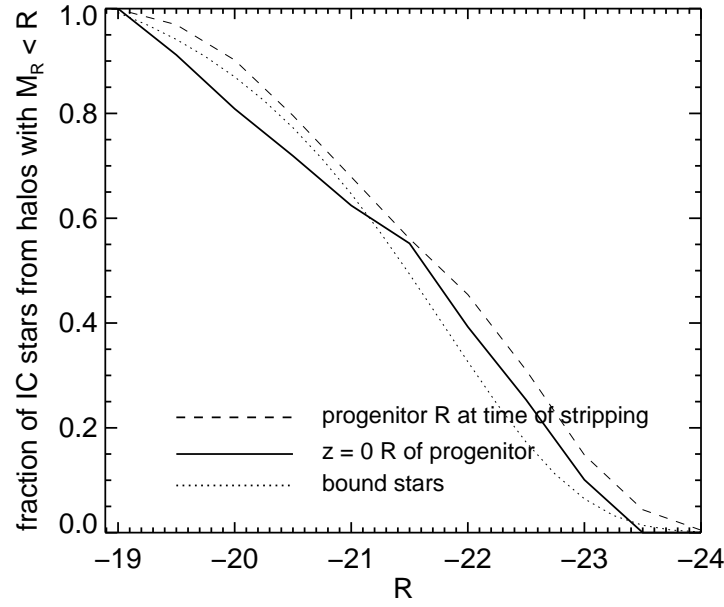

Figure 6. The fraction of intracluster stars stripped from halos as a function of their progenitor's absolute magnitude, both at the time of stripping and traced forward to $\mathrm{z}=0$. The fraction of stars bound to halos with $\mathrm{M}_{R}<R$ is also overplotted.

Stars in the outskirts of $\mathrm{L}^{*}$ and brighter galaxies have photometric properties that differ from those of the stars producing the majority of galaxy light, due to negative metallicity gradients (Peletier et al. 1990). Therefore, stars stripped from the outer regions of the most massive galaxies could have the same colors and metallicities of an 'average' star in an intermediate luminosity galaxy. For example, the outer regions of elliptical galaxies have been observed to have (B-R) that are $\sim 0.1-0.15$ magnitudes bluer than the inner regions (Peletier et al. 1990). Gladders et al. (1998)'s observations of the red sequences of galaxy clusters showed that the average $(B-R)$ of dwarf galaxies are $\sim 0.3$ magnitudes bluer than the average (B-R) of the most luminous cluster galaxies. Therefore, we expect many IC stars to have colors in common with intermediate luminosity galaxies (redder than the dwarfs, bluer than the most luminous), although they have been stripped from galaxies brighter than $\mathrm{M}^{*}$. This prediction is consistent with the Durrell et al. (2002) measurement of $-0.8<[\mathrm{Fe} / \mathrm{H}]<-0.2$ for the range of metallicities of intracluster stars in Virgo. This metallicity range agrees with the mean metallicities of intermediate luminosity galaxies.

Low surface brightness (LSB) and dwarf galaxies fall below the resolution limit of our simulation. We thus likely underestimate the fraction of unbound stars. Could LSBs and dwarfs actually be the population contributing most of the IC stars? Durrell et al. (2002) presented the example, that although dwarf galaxies contribute only $\sim 10 \%$ of the light of Virgo, the IC stars contain $\sim 20 \%$ of the light in Virgo. This means that for the IC stars to have all come from dwarfs, the number of dwarfs must have once been 3 times their present number. This extreme scenario is not required, as our results show that massive galaxies produce substantial ICL. Simulations that include galaxies with a more realistic distribution of profiles and/or include galaxies even further down the luminosity function are necessary to know the stripping efficiency as a function of luminosity more precisely than we present here. 


\section{INTRACLUSTER STARS AS TRACERS OF DYNAMICAL HISTORY}

In $\S 3$, we showed that the cluster is dynamically unrelaxed and has accumulated a substantial fraction of its IC population over the last few Gyrs. In this section, we investigate the extent to which this dynamical history is reflected in the observable spatial and velocity distributions of the cluster's stars.

\subsection{Spatial Distribution}

Gregg \& West (1998) and Calcáneo-Roldán et al. (2000) identified cold stellar streams in Coma with $\mu_{R} \sim 26.0$. Such streams are evidence for recent stripping events, because stars have not had sufficient time to get mixed into the global cluster potential. C2 underwent several major stripping events within the last $2 \mathrm{Gyr}$, associated with the infall of a Virgo sized group ('Group One' in Figure 1). As a result, the cluster exhibits a wide stream of unbound stars that extends for hundreds of $\mathrm{kpc}$ between the $\mathrm{cD}$ galaxy and Group One, along the line that it recently crossed the $\mathrm{cD}$. The average surface brightness of the unbound stars in this large feature is $\sim \mu_{R}=26.5$. We find no other streams in $\mathrm{C} 2$, but we likely underestimate the prevalence of streams that result from such an accretion event, because the galaxies in this simulation are all spheroidal. Therefore, they all produce streams with internal velocity dispersions $\left(\sigma_{\text {stream }}\right)$ that are comparable to $\mathrm{v}_{\text {rot }}$, rather than the much colder streams produced by disk galaxies $\left(\sigma_{\text {stream }} \sim \sigma_{\text {disk }}\right)$.

The surface brightness profile of C2's IC stars is in agreement with that observed in previous studies of both Coma and of other Abell clusters. Figure 7 displays the azimuthally averaged surface density profiles of: the galaxy cluster (all stars+dark matter), the stars bound to individual galaxies, and the intracluster stars. The profiles have been normalized to the average surface density of the cluster within the central $100 \mathrm{kpc}$. Although the recent merger event results in an asymmetric IC star distribution, their azimuthally averaged surface density profile beyond $250 \mathrm{kpc}$ follows that of the galaxies, as also seen in two of the Abell clusters observed by Feldmeier et al. (2004). Within 250 $\mathrm{kpc}$, IC stars have a flatter profile than bound stars (including cD stars), at difference with the results of Murante et al. (2004). In fact, our simulated IC population has a nearly flat surface brightness profiles between 50 and $250 \mathrm{kpc}$. Beyond $50 \mathrm{kpc}$, most of the light in the bound stellar profile is not from $\mathrm{cD}$ stars. The average R-band surface brightness of intracluster stars $250 \mathrm{kpc}$ from C2's center is 26.2, which agrees with that measured for Coma by Bernstein et al. (1995).

Figure 8 shows the azimuthally averaged total projected stellar profile of the $\mathrm{cD}$ galaxy with 2 de Vaucouleurs fits overplotted. The profile is inconsistent with a typical cD profile of a single $\mathrm{R}^{1 / 4}$ law, either with or without an excess at large radii. The unusually steep slope in the inner 25 $\mathrm{kpc}$ is likely due to the unrealistic cooling flow and ongoing star formation at the center of our $\mathrm{cD}$, and is not seen in the profiles of the other simulation galaxies. Although the radius ranges fit are arbitrary, the fits highlight a true flattening in the outer envelope of the $\mathrm{cD}$ galaxy, around $200 \mathrm{kpc}$. This flattening can not directly be due to Group One or Two, as

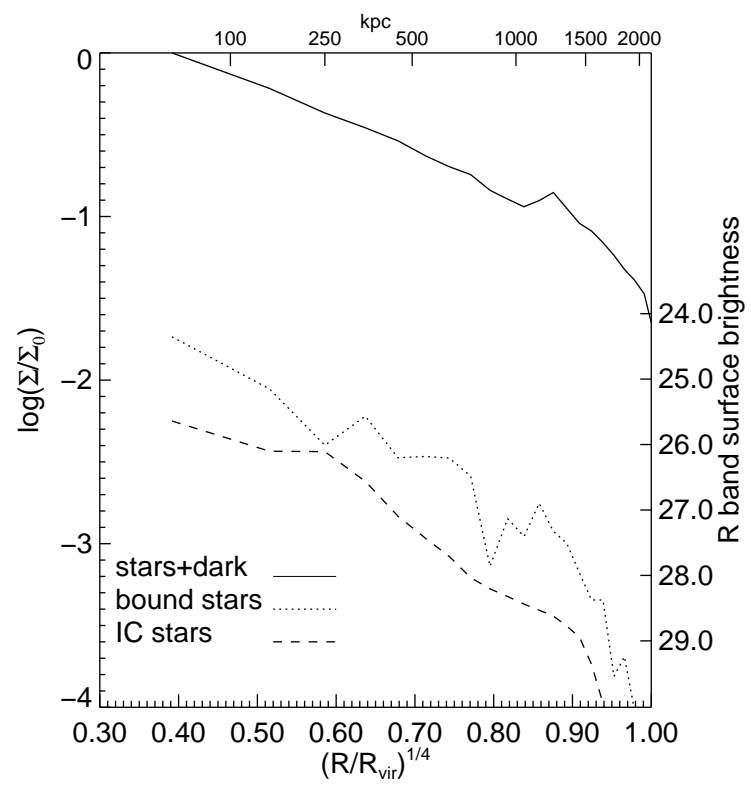

Figure 7. Comparison of the surface density profiles of: the stars bound to galaxies, the intracluster stars, and all stars + dark matter. These projected profiles only include stars within the virial radius of the galaxy cluster.

they are a Mpc from the cluster center. This excess above a $R^{1 / 4}$ profile is similar to that seen by Feldmeier et al. (2002) for the outer profile $(>80 \mathrm{kpc}$ ) of the $\mathrm{cD}$ galaxy in the rich galaxy cluster A1413. There is building evidence that such envelopes may even be ubiquitous features of $\mathrm{cD}$ galaxies of rich clusters (Gonzalez et al., in preparation). The fact that C2's cD displays an excess over $\mathrm{R}^{1 / 4}$ at large $\mathrm{R}$ supports Feldmeier et al. (2002)'s suggestion that such envelopes are a signature of recent cluster growth. An intracluster population formed primarily during the initial cluster collapse differs from that of an intracluster population accumulated slowly over time via tidal processes, and is expected to follow an $\mathrm{R}^{1 / 4}$ out to even larger radii than seen around C2 (Merritt 1984; Dubinski 1998).

\subsection{Substructure in Velocity Space}

We looked at the phase-space distribution of "planetary nebulae" (PNe) in four $120 \times 120 \mathrm{kpc}$ fields $\left(30^{\prime} \times 30^{\prime}\right.$ at a distance of $15 \mathrm{Mpc}$ ), selected by eye to lie outside the cluster galaxies. We used the luminosity-specific Planetary Nebula number density of $\alpha_{1.0}=9.4 \times 10^{-9} \mathrm{PNe} \mathrm{L}_{\odot}^{-1}$ Ciardullo et al. 1989) to randomly select a number of stars in each field representative of the number of Planetary Nebulae that existing spectroscopic surveys would observe.

Figure 9 shows the observable phase space distributions and line-of-sight velocity histograms of PNe in the two of the four fields plotted on Figure 1 The fact that these 'observed' fields display both visible phase space correlations and nonGaussian distributions of line of sight velocities reflects the young dynamical age of the IC population, also found by Napolitano et al. (2003) when they applied the same analysis to their simulations. The small scale phase-space sub- 

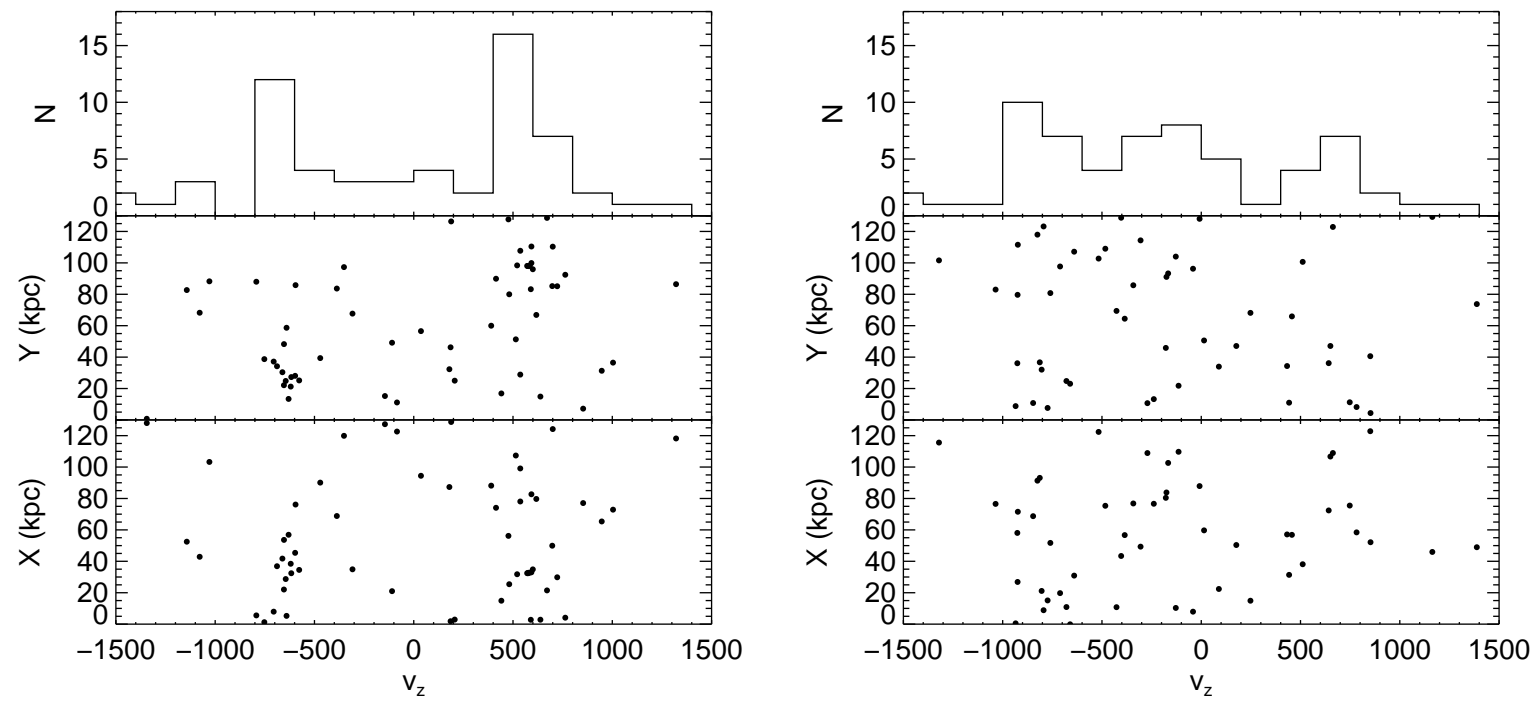

Figure 9. The spatial and velocity distribution of stars in the 2 'empty' fields located $500 \mathrm{kpc}$ from the cluster center (shown in Figure 1 .

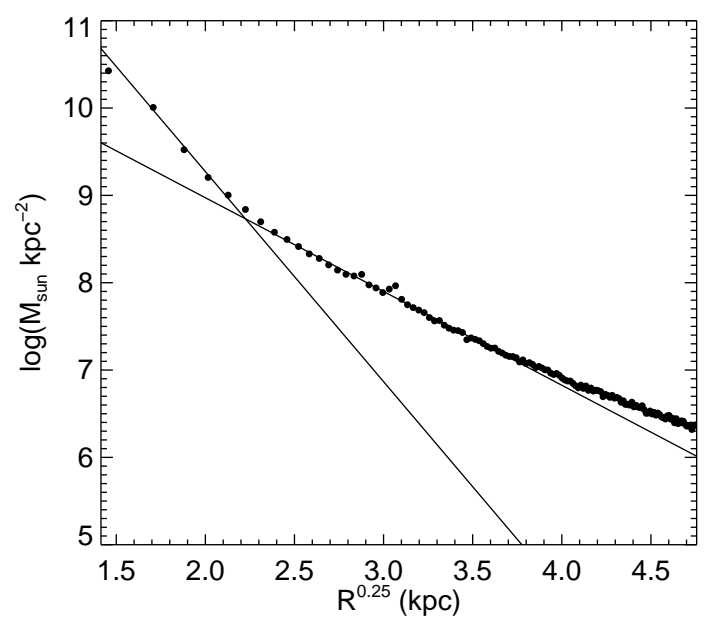

Figure 8. Surface density profile of the $\mathrm{cD}$ galaxy. Two $\mathrm{R}^{1 / 4}$ fits are overplotted; one was fit from $5-20 \mathrm{kpc}$ and one was fit from $40-200 \mathrm{kpc}$.

structure seen in our cluster's stars represents a lower limit on the actual substructure one would expect to observe, because all of the galaxies in our cluster are elliptical, so stellar streams have higher internal velocity dispersions, on average, and are thus more easily mixed than those that would result from a population that contains disk galaxies.

\subsection{The Anisotropy Profile of the IC Stars}

The top panel of Figure 10 shows the 1D radial profiles of the line-of-sight (los) velocity dispersions of IC stars, cluster galaxies, and cluster dark matter particles. To derive the error bars, we did a bootstrap resampling of the data for numerous cluster orientations. We find that the intracluster stars have a velocity dispersion on average $\sim 20 \%$ smaller than that of either the galaxies or the dark matter. The IC stars' velocity dispersion decreases with radius a bit more than that of the dark matter or the galaxies. This difference in velocity profile is due to the fact that, beyond $750 \mathrm{kpc}$, the intracluster star orbits are more radial than those of either the dark matter or the galaxies. This bias is seen in the comparison between the anisotropy profiles $\left(\beta=1-<\sigma_{t}^{2}>/<\sigma_{r}^{2}>\right)$ of IC stars, galaxies, and dark matter in the lower panel of Figure 10 Although Sommer-Larsen et al. (2004) did not find such an anisotropy bias in their simulations, it is not unexpected for unbound stars to have more radial orbits than galaxies or dark matter; stars are preferentially stripped from galaxies on radial orbits because, on average, such orbits have smaller pericenters than isotropic orbits (Taffoni et al. 2003). This bias in the anisotropy of intracluster stars, relative to that of either cluster galaxies or the dark matter, needs to be considered when constructing dynamical models based on intracluster stellar tracers. In the next section, we will do a simple calculation to demonstrate the possible effect of this difference on cluster mass estimates.

\section{INTRACLUSTER STARS AS A TRACER OF CLUSTER MASS}

The number of intracluster PNe easily surpass that of cluster galaxies, which suggests that $\mathrm{PNe}$ may be a more effective tracer of the cluster mass distribution than galaxies (Ford et al. 2002). Modeling the mass of a system based on the observed light and line of sight velocity dispersion profile relies on assumptions about either the mass distribution or the distribution of orbits. Therefore, the extent to which free floating stars can be used as mass tracers is determined by the extent to which we understand these properties of the intracluster population relative to that of the underlying mass distribution.

In $\S 4.2$, we showed that IC stars are correlated in phase 


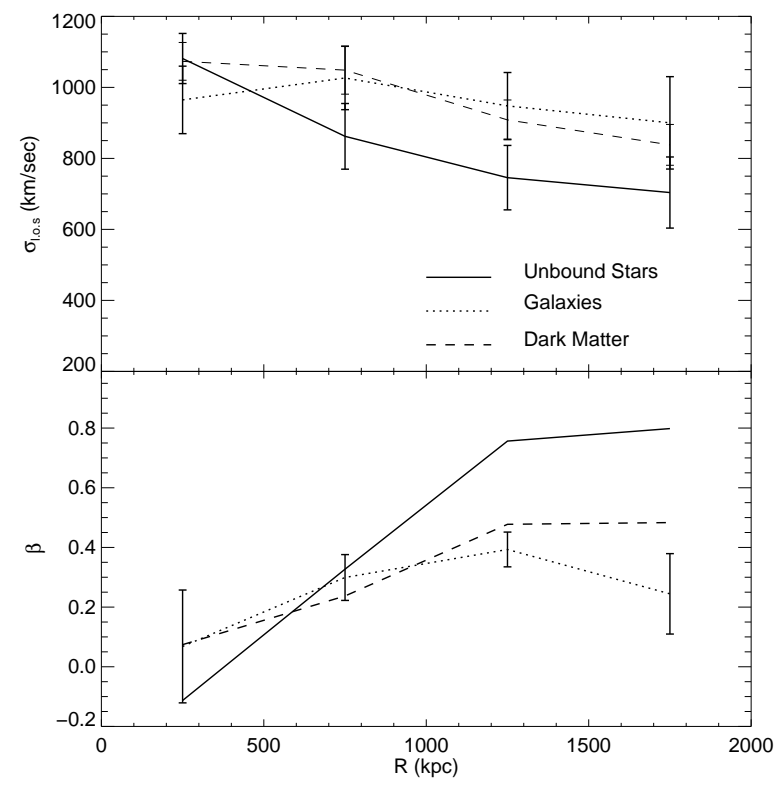

Figure 10. Top panel: The line-of-sight velocity dispersion profiles of the intracluster stars, the dark matter, and the cluster galaxies. $\beta=-\infty$ corresponds to circular orbits, $=0$ corresponds to isotropic orbits, and $=1$ corresponds to radial orbits. Bottom panel: The 3D anisotropy profile. Cluster projection does not affect the 3D $\beta$ profile. Error bars are not plotted for dark matter and unbound stars, because the formal bootstrapped errors are tiny, due to the large number of data points in each bin.

space and have a mean orbital distribution that differs from that of the cluster galaxies and dark matter. Furthermore, samples of galaxy cluster PNe are identified in discrete fields, such as those of Arnaboldi et al. (2002) and Feldmeier et al. (1998). In this section, we use a crude approach to explore if PNe may nonetheless be useful tracers of cluster mass.

The "projected virial theorem" is one simple dynamical method typically used to derive galaxy cluster masses (Girardi et al. 1998). However, numerous close pairs of objects will be present in samples of Planetary Nebulae identified in discrete fields, resulting in a projected virial mass that is more than an order of magnitude less than the actual cluster mass. We therefore use the "projected mass" to evaluate Planetary Nebulae as mass tracers, because it is less sensitive to close pairs of objects (Rines et al. 2003). We computed the projected mass $(\mathrm{PM})$ with the relation from Heisler et al. (1985):

$M_{V}=\frac{32}{\pi G} \frac{R_{i}\left(v_{i}-v\right)^{2}}{N}$

that assumes isotropic orbits and a continuous mass distribution (Rines et al. 2003). The factor of 32 becomes 16 or 64 for circular or radial orbits, respectively.

First, we calculated the projected mass with $\mathrm{PNe}$ from a number of $30^{\prime} \times 30^{\prime}$ fields ranging from $1-14$, and with a spectroscopic depth of either the brightest 0.5 or 1.0 magnitudes of the PNLF. We randomly placed 40 fields at distances between 0.5 and $1.0 \mathrm{Mpc}$ from the cluster center and automatically selected those fields farthest from any galaxy. The projected mass was thus calculated for numerous cluster projections. The average mass as a function of number

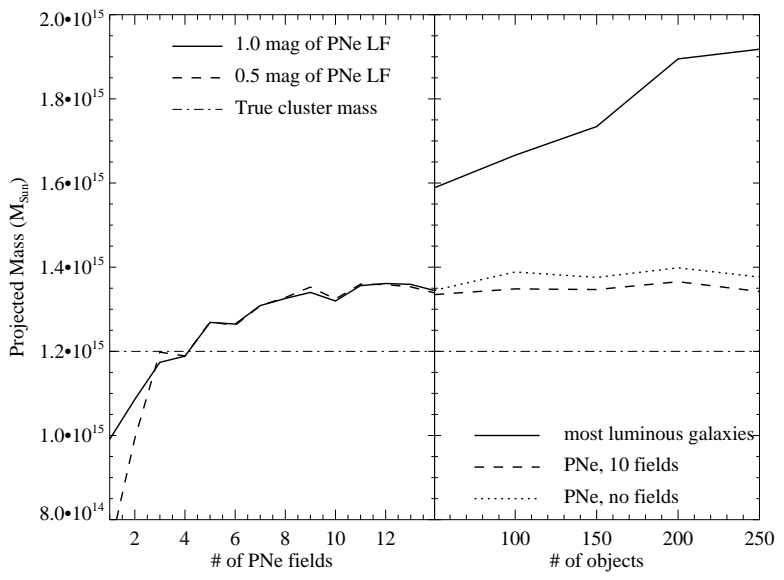

Figure 11. Left panel: Projected mass of the galaxy cluster calculated using a simulated sample of Planetary Nebulae selected in discrete fields between a projected radius of 500 and $1000 \mathrm{kpc}$, using either the brightest 0.5 mag or the brightest 1.0 mag in each of up to 14 fields. Right panel: Projected mass calculated using the $\mathrm{N}$ brightest galaxies, $\mathrm{N}$ randomly selected unbound stars, or $\mathrm{N}$ simulated Planetary Nebulae distributed between $140.5 \times 0.5$ degree $^{2}$ fields.

of fields is shown in the left panel of Figure 11 This plot shows that the number of $\mathrm{PNe}$ fields used in the analysis is more important than the depth of the spectroscopic follow up, for purposes of calculating mass. The scatter due to cluster projection is $\sim 25 \%$ of the total cluster mass, similar to the dispersion found by Governato et al. (2001). Varying the clustercentric radii of the $\mathrm{PNe}$ fields showed that fields at a wide range of radii need to be included to avoid over/underestimates on the order of $20 \%$ the mean calculated value. Coma is larger than Virgo, so it may be that PNe in fields such as Arnaboldi et al. (2002)'s RCN1 and fields 2 and 6 of Feldmeier et al. (2003) are at sufficiently large radii to be included in an accurate mass measurement.

The projected mass calculated with 5 or more fields nearly asymptotes to the value calculated using unbound stars not restricted to individual fields $\left(1.4 \cdot 10^{15} M_{\odot}\right)$, shown in the right panel of Figure 11 Surprisingly, this calculated $\mathrm{PM}$ is within $10 \%$ of the actual cluster mass of $1.5 \cdot 10^{15}$. This result is initially surprising because Figure 10 shows both that IC stars have a velocity dispersion that is somewhat smaller than that of the underlying mass distribution and that IC stars do not tend to follow isotropic orbits. These factors produce a mass underestimate relative to an isotropic population with the same velocity dispersion as the underlying mass distribution, when using Equation 1. However, this underestimate is canceled out by the fact that Equation 1 systematically produces an overestimate of mass relative to true cluster mass, similar to the overestimate produced by the projected virial theorem without a surface term correction (Aceves \& Perea 1999; Rines et al. 2003). We found this same overestimate when we calculated the average projected mass with C2 cluster galaxies, as seen in the right panel of Figure 11 The $1 \sigma$ scatter due to cluster projection is $\sim 15 \%$ for the masses calculated using galaxies. We also found that the projected masses calculated using galaxies are very sim- 
ilar to the projected virial masses without a correction for the surface term.

Our analysis shows that IC PNe are worth a closer investigation into their utility in making detailed mass models of nearby clusters. The fact that our analysis yielded a cluster mass very close to the true mass was partially a fortuitous canceling of two effects; a detailed analysis would thus likely produce a more reliable approach to cluster mass modeling with individual IC stars than we have presented here.

\section{CONCLUSION}

In this paper, we used a high-resolution, SPH simulation of a Coma-like cluster formed in a cosmological context to study the formation, evolution and properties of intracluster stars in a rich galaxy cluster. This simulation resolves galaxies as faint as $M_{R}=-19$, which includes over $85 \%$ of the light from cluster galaxies. Furthermore, the simulated stellar distributions have a range of effective radii that matches that observed by Bernardi et al. (2003). This dynamic range and accurate stellar distribution are essential for relying on simulated stars as accurate tracers of the dynamically stripped stellar population.

Overall, we find that the fraction and distribution of IC stars in a hierarchical scenario are a good match to the observed properties, qualitatively similar to the results found in the lower resolution simulations of Murante et al. (2004) and Sommer-Larsen et al. (2004). ICL formation is an ubiquitous, ongoing process, with an unbound stars fraction that slowly increases with time. However, the IC star fraction at any given time is not clearly correlated with the dynamical state of the cluster, with both the lowest and highest IC fractions occurring after substantial merging events. The intracluster population is formed via both stripping within the cluster and via the infall of large groups that contain stars that are already unbound. As a result, there is a link between the accumulation of IC stars and infall into the cluster.

Our analysis traced the fraction of stars stripped from galaxies as a function of galaxy luminosity. We found that the most luminous galaxies in a cluster contribute a substantial fraction of stars to the unbound population, and that the stripping efficiency is roughly constant over a wide range of galaxy masses. As a result, we predict that IC stars will have photometric properties in common with the average properties of intermediate luminosity galaxies, which is consistent with existing observations (e.g. Durrell et al 2002).

We verified the phase space and velocity distribution of IC stars found in previous numerical work. IC stars exhibit non-Gaussian velocity distributions, as well as visible correlations in their observable phase space plots (Napolitano et al. 2003). We also showed that the orbits of IC stars are more radially biased than those of the overall galaxy or dark matter, which results in the IC stars having a lower l.o.s. velocity dispersion in the outskirts of the cluster than in its inner regions.

We find that despite the fact that IC stars do exhibit phase space substructure, and have l.o.s. velocities and anisotropies that differ from that of the underlying distribution, they are promising tracers of galaxy cluster mass.
The depth of the spectroscopic observations in each field makes little difference in the calculated masses. The projected masses calculated with discrete fields of unbound stars quickly approaches that calculated with randomly selected unbound stars, which is within $10 \%$ of the true cluster mass due to a fortuitous canceling of two effects. Our analysis suggests that more detailed analysis may provide a "correction" to the projected mass found with IC stars, similar to the known correction for the projected virial mass estimate.

Much progress remains to be done to complete our understanding of intracluster stars and what they can tell us about the formation history of the clusters which they inhabit. Some groups are already (e.g. Mihos et al 2004) starting to include galaxies with more realistic profiles into numerical simulations, which will enable a more quantitative analysis of substructure predictions and of stripping efficiencies. Better resolution coupled with a more realistic implementation of feedback should be the goal of future cosmological simulations used to study the properties of intracluster stars. These improvements will enable more robust predictions of colors, gradients and light profiles than possible with the current generation of simulations.

\section{ACKNOWLEDGMENTS}

We would like to thank Anthony Gonzalez, Andrey Kravtsov, and Japser Sommer-Larsen for helpful comments on this manuscript. BW would like to thank George Lake for helpful conversations during this project. BW would like to acknowledge support from the UW Royalty Research Fund, NSF Grant AST-0098557, and NSF grant AST-0205413. FG is a Brooks Fellow and was supported in part by NSF grant AST-0098557. TRQ acknowledges support from NSF grant AST-0098557 and NSF grant AST-0205413. The simulations were run at the Arctic Region Supercomputing Center.

\section{REFERENCES}

Aceves H., Perea J., 1999, A\&A, 345, 439

Arnaboldi M., Aguerri J. A. L., Napolitano N. R., Gerhard O., Freeman K. C., Feldmeier J., Capaccioli M., Kudritzki R. P., Méndez R. H., 2002, AJ, 123, 760

Arnaboldi M. et al, 2003, AJ, 125, 514

Balogh M. L., Pearce F. R., Bower R. G., Kay S. T., 2001, MNRAS, 326, 1228

Bell E. F., de Jong R. S., 2001, ApJ, 550, 212

Bell E. F., McIntosh D. H., Katz N., Weinberg M. D., 2003, ApJS, 149, 289

Bernardi M. et al, AJ, 125, 1849

Bernstein G. M., Nichol R. C., Tyson J. A., Ulmer M. P., Wittman D., 1995, AJ, 110, 1507

Borgani S., Governato F., Wadsley J., Menci N., Tozzi P., Quinn T., Stadel J., Lake G., 2002, MNRAS, 336, 409

Calcáneo-Roldán C., Moore B., Bland-Hawthorn J., Malin D., Sadler E. M., 2000, MNRAS, 314, 324

Ciardullo R., Jacoby G. H., Ford H. C., Neill J. D., 1989, ApJ, 339, 53

Cole S., Lacey C. G., Baugh C. M., Frenk C. S., 2000, MNRAS, 319, 168

Cole S. et al. , 2001, MNRAS, 326, 255 
Diemand J., Moore B., Stadel J., Kazantzidis S., 2004, MNRAS, 348, 977

Dubinski J., 1998, ApJ, 502, 141

Durrell P. R., Ciardullo R., Feldmeier J. J., Jacoby G. H., Sigurdsson S., 2002, ApJ, 570, 119

Feldmeier J. J., Ciardullo R., Jacoby G. H., 1998, ApJ, 503, 109

Feldmeier J. J., Ciardullo R., Jacoby G. H., Durrell P. R., 2003, ApJS, 145, 65

Feldmeier J. J., et al., 2004, astro-ph/0403414

Feldmeier J. J., Mihos J. C., Morrison H. L., Rodney S. A., Harding P., 2002, ApJ, 575, 779

Ford H., Peng E., Freeman K., 2002, in ASP Conf. Ser. 273:

The Dynamics, Structure \& History of Galaxies: A Workshop in Honour of Professor Ken Freeman Extragalactic Planetary Nebulae.

Gal-Yam A., Maoz D., Guhathakurta P., Filippenko A. V., 2003, AJ, 125, 1087

Ghigna S., Moore B., Governato F., Lake G., Quinn T., Stadel J., 1998, MNRAS, 300, 146

Girardi M., Giuricin G., Mardirossian F., Mezzetti M., Boschin W., 1998, ApJ, 505, 74

Gladders M. D., Lopez-Cruz O., Yee H. K. C., Kodama T., 1998, ApJ, 501, 571

Gonzalez A. H., Zabludoff A. I., Zaritsky D., Dalcanton J. J., 2000, ApJ, 536, 561

Gonzalez A. et al, 2004, in preparation

Governato F., Ghigna S., Moore B., Quinn T., Stadel J., Lake G., 2001, ApJ, 547, 555

Gregg M. D., West M. J., 1998, Nature, 396, 549

Haardt F., Madau P., 1996, ApJ, 461, 20

Heisler J., Tremaine S., Bahcall J. N., 1985, ApJ, 298, 8

Hughes J. P., 1989, ApJ, 337, 21

Katz N., Weinberg D. H., Hernquist L., 1996, ApJS, 105, 19

Katz N., White S. D. M., 1993, ApJ, 412, 455

Krick J. et al, 2004, in preparation

Mayer L., Governato F., Colpi M., Moore B., Quinn T., Wadsley J., Stadel J., Lake G., 2001, ApJ, 547, L123

Merritt D., 1984, ApJ, 276, 26

Mihos C. et al, 2004, in preparation

Mobasher B., Colless M., Carter D., Poggianti B. M., Bridges T. J., Kranz K., Komiyama Y., Kashikawa N., Yagi M., Okamura S., 2003, ApJ, 587, 605

Moore B., Katz N., Lake G., Dressler A., Oemler A., 1996, Nature, 379, 613

Murante G., et al., 2004, astro-ph/0404025 ApJL accepted Napolitano N. R., Pannella M., Arnaboldi M., Gerhard O., Aguerri J. A. L., Freeman K. C., Capaccioli M., Ghigna S., Governato F., Quinn T., Stadel J., 2003, ApJ, 594, 172 Okamura S. et al. , 2002, PASJ, 54, 883

Peletier R. F., Davies R. L., Illingworth G. D., Davis L. E., Cawson M., 1990, AJ, 100, 1091

Power C., Navarro J. F., Jenkins A., Frenk C. S., White S. D. M., Springel V., Stadel J., Quinn T., 2003, MNRAS, 338,14

Rines K., Geller M. J., Kurtz M. J., Diaferio A., 2003, AJ, 126, 2152

Secker J., Harris W. E., Plummer J. D., 1997, PASP, 109, 1377

Sommer-Larsen J., Romeo A. D., Portinari L., 2004, astro-ph/0403282 MNRAS submitted
Springel V., Hernquist L., 2003, MNRAS, 339, 312

Taffoni G., Mayer L., Colpi M., Governato F., 2003, MNRAS, 341, 434

Theuns T., Warren S. J., 1997, MNRAS, 284, L11

Trentham N., Mobasher B., 1998, MNRAS, 293, 53

Wadsley J. W., Stadel J., Quinn T., 2004, New Astronomy, 9, 137

Zibetti S., White S. D. M., 2004, astro-ph/0404326

Zwicky F., 1951, PASP, 63, 61 